\section{Handbook of Breast MRI}

J. Price

Cambridge, U.K.: Cambridge University Press, 2011, 213 pages, $\$ 80$

Breast MRI has firmly established its place as a mainstream imaging technique in the last decade, particularly in the screening of high-risk women and in the preoperative assessment of patients with known breast cancer. The role of breast MRI in the screening of high-risk women is now unchallenged. With high-gradientstrength MRI instruments and technical advances in breast coil design, image quality has improved immeasurably, and computeraided detection systems make the task of reporting breast MRI studies much quicker and easier. Gene expression profiling has provided important new insights into the pathogenesis of heterogeneous breast cancer and may be correlated with MRI findings. This book aims to assist radiologists seeking to add breast MRI to their skill set for the better management of breast cancer.

This book is organized into 8 chapters, starting with introductory chapters (basics, anatomy, and pathology) and continuing with detailed clinical chapters (interpretation, biopsy, screening, staging, problem-solving applications, and MRI after breast augmentation). Each chapter begins with an outline and ends with multiple-choice questions. The images in the 76 figures are illustrative, and the tables are useful. The references are updated, and there are unique appendices dealing with nephrogenic systemic fibrosis, the sensitivity and specificity of diagnostic tests, the TNM classification, an overview of surgical procedures, radiation therapy and systemic therapy, preventive strategies, and genes and genetic testing.

This book provides core knowledge and clinical guidelines for performing breast MRI in everyday practice and is an invaluable practical diagnostic resource for radiologists, breast surgeons, oncologists, and any clinicians involved in breast cancer management. Trainees in radiology, surgical oncology, and medical oncology will also benefit from this book.

\section{E. Edmund Kim}

University of California at Irvine

101 The City Dr. S.

Orange, CA 92868

E-mail: edmundek@uci.edu

Published online Nov. 12, 2012.

DOI: 10.2967/jnumed.112.112417

\section{Pearls and Pitfalls in Thoracic Imaging: Variants and Other Difficult Diagnoses}

\author{
T. Hartman, ed.
}

Cambridge, U.K.: Cambridge University Press, 2011, 224 pages, \$99

Differentiation of normal variants from rare diseases may be difficult on thoracic imaging, and a really concise book on that topic is lacking. Therefore, this book was designed to fill that niche, giving guidance for identifying normal variants that can be ignored and showing pathognomonic images of rare diseases. However, this is not a book of exhaustive differentials for nonspecific findings or a book to teach how to interpret common disease findings on chest CT.

The 12 sections comprise 86 cases ( 9 of the airways, 17 of the lung parenchyma, 8 of the mediastinum, 8 of the esophagus, 9 of the aorta, 10 of the vessels, 4 of the pericardium, 4 of the pleura, 2 of the diaphragm, 3 of the lymphatics, 7 involving PET/CT, and 5 involving artifacts). Each case has a descriptive image, a discussion of the importance of the case and the typical clinical scenario, and a differential diagnosis. The teaching point of the case is highlighted before a list of recent references is presented. The images in the 212 figures are excellent and illustrative.

The image-rich text is laid out such that a reader facing a potential abnormality simply locates the section of the book that covers the pertinent region and then looks for an image in that section that matches the finding. Once a matching image is found, the reader can refer to the heading to see what the diagnosis is. Written by leading thoracic radiologists with concise descriptions, the book enables identification of normal variants and provides pathognomonic images of rare diseases to help make that one-ina-million diagnosis.

This book is for the practicing radiologist or radiology resident who is reviewing an examination and has a specific question about a normal variant or about an abnormal finding that may be due to an artifact or rare disease. Trainees and practitioners in thoracic medicine and surgery will also benefit from this book.

\author{
E. Edmund Kim \\ University of California at Irvine \\ 101 The City Dr. S. \\ Orange, CA 92868 \\ E-mail: edmundek@uci.edu
}

COPYRIGHT @ 2013 by the Society of Nuclear Medicine and Molecular Imaging, Inc.

Published online Nov. 12, 2012. DOI: 10.2967/jnumed.112.112409 